\title{
MODELLING OF SHIP'S TRAJECTORY PLANNING IN COLLISION SITUATIONS BY HYBRID GENETIC ALGORITHM
}

\author{
Shengke Ni \\ Zhengjiang Liu \\ Yao Cai \\ Xin Wang \\ Dalian Maritime University, China
}

\begin{abstract}
Ship collision-avoidance trajectory planning aims at searching for a theoretical safe-critical trajectory in accordance with COLREGs and good seamanship. In this paper, a novel optimal trajectory planning based on hybrid genetic algorithm is presented for ship collision avoidance in the open sea. The proposed formulation is established based on the theory of the Multiple Genetic Algorithm (MPGA) and Nonlinear Programming, which not only overcomes the inherent deficiency of the Genetic Algorithm (GA) for premature convergence, but also guarantees the practicality and consistency of the optimal trajectory. Meanwhile, the encounter type as well as the obligation of collision avoidance is determined according to COLREGs, which is then considered as the restricted condition for the operation of population initialization. Finally, this trajectory planning model is evaluated with a set of test cases simulating various traffic scenarios to demonstrate the feasibility and superiority of the optimal trajectory.
\end{abstract}

Keywords: trajectory planning, Multiple Genetic Algorithm, ship collision avoidance, nonlinear programming, COLREGs

\section{INTRODUCTION}

Currently, the navigation of ships is mainly depending on the navigators' experience and techniques coupled with traditional practice of good seamanship. With the growing overload, safety issues associated with human errors contribute to about 75 percent of maritime accidents and lead to significant human, monetary and environmental loss [12]. Therefore, unmanned surface vehicles have been gaining attention in the past decade. For example, in 2005, e-navigation was an initiative started by IMO to increase the safety of navigation by using modern technology. In 2012, the European Union invested in the MUNIN (Maritime Unmanned Navigation through Intelligence in Networks) project to develop an autonomous dry bulk carrier [3]. In 2015, the Finnish Funding Agency approved the academic research project - Advanced Autonomous Waterborne Applications
(AAWA). In 2016, Rolls-Royce published the white paper to announce their plan for the construction of autonomous ship [9].

One of the key points for the autonomous ship formulation is to construct the Decision Support System. And the main challenges are to find the optimum approaches in the aspects of the situational awareness and the trajectory planning. Currently, the technologies, in particular navigational instruments such as Automatic Identification System (AIS), Automatic Radar Plotting Aid (ARPA) and Electronic Chart Display and Information System (ECDIS) etc., have supplied a revolutionary platform for the former, thus the selecting of an appropriate algorithm for determining the safe trajectory becomes an urgent matter. In fact, the development of ship's trajectory planning will be a gradual and iterative process subject to extensive testing and simulation. It is remarkable that the evasive manoeuvres in the optimal trajectory should 
be based on an interpretation of common practice, which performs the process of collision avoidance with fewer course deviations.

On topic of ship's trajectory planning, a number of studies have been performed and reviewed $[18,26]$. For example, the existing approaches cover the Sequential Gradient Restoration Algorithm [14], Recursive Algorithm [5], Fuzzy Set Theory [6-7], Knowledge-based System [11], the Interval Programming [2], the Maze Routing Algorithm [21], the Fast Marching Method [13], Cooperative Path Planning Algorithm [29], Distributed Decision Support Formulation [35], the Artificial Potential Field [34], the Fuzzy Logic based method [15-16], Genetic Algorithm [8, 31, 32], Evolutionary Algorithm $[17,22,23,24,25,28]$, the Ant Colony Algorithm [10, 30], the Danger Immune Algorithm [33]. Based on the methods, the approaches assume that ship's trajectory planning can be categorized into two general groups, namely the deterministic and heuristic ones [26]. The deterministic approaches follow a set of rigorously defined steps to converge to the feasible solutions characterized by low computational time, but they might be incapable of solving complex situations. The heuristic approaches possess the ability to deal with complex environment, and it only searches inside a subspace of the search space for an optimal solution that satisfies the design requirements. However, the optimal evasive manoeuvre usually involves multiple course alterations which is not complaint with the common practice. In addition, the consistency of the heuristic approaches' outputs cannot be guaranteed, which hinders its adoption for application.

In recent years, EA and GA have received great concern by scholars in the field of trajectory planning. They are both the subset of evolutionary computation and are mainly different in the evolutionary manners. In fact, EA is a next generation of GA. These techniques, based on the important principle of 'survival of the fittest', model some natural phenomena of genetic and phenotypic inheritance and Darwinian strife for survival. It is remarkable that GA is easy to combine with other algorithms to improve its optimal ability. However, the previous work based on GA mainly employed the Standard Genetic Algorithm (SGA) to search for a satisfactory trajectory for ship collision avoidance. Before the optimization, it needs to debug the parameters'values for different genetic operators by experiment.

In the light of the above given comments, this paper modifies the algorithm by combining the Multiple Genetic Algorithm (MPGA) and the Nonlinear Programming methodology. The idea of the MPGA is that several populations with different combinations of parameters' value for genetic operators evolve simultaneously. Meanwhile, these populations are related to each other by the establishment of Immigrant Operator and Elite-individual Operator, which guarantee the information exchange and the cooperative work among populations. As a result, the MPGA solves the problem of parameters' setting and is beneficial for improving the ability of local optimization. In order to further enhance the ability of local optimization and ensure the practicality and consistency of the optimal trajectory, the Nonlinear
Programming methodology is incorporated into the MPGA at the specific generation. Additionally, the proposed algorithm adopts a real number encoding method which is convenient for handling constraint conditions. All the simulations are based on the assumption that the own ship is the only manoeuvring party, and target ships keep their heading and speed.

This article is composed of seven sections. Section 1 briefly introduces the motivation behind the study. Section 2 explains the division of the encounter situation. Section 3 provides an overview of the SGA and MPGA. Section 4 illustrates the local optimal algorithm - Nonlinear Programming. Section 5 describes this trajectory planning model-the hybrid genetic algorithm. Section 6 outlines the test cases used to discuss the feasibility and superiority of this trajectory planning model. This study is then summarized in section 7 .

\section{JUDGMENT OF THE ENCOUNTER SITUATION}

In fact, only when the encounter type and the obligation of collision avoidance are classified, the calculation of trajectory planning for collision avoidance in marine traffic can be conducted. Each target ship is categorized into a particular encounter type based on its position, heading as well as its relative bearing with respect to the heading of the own ship (OS).

The coordinate system $X O Y$ is established as shown in Fig. 1. Let's assume the coordinate, speed and heading of the OS are $\left(X_{O}, Y_{O}\right), V_{\mathrm{O}}$ and $\varphi_{\mathrm{O}}$, and the corresponding information of the target ship (TS) are $\left(X_{\mathrm{T}}, Y_{\mathrm{T}}\right), V_{\mathrm{T}}$ and $\varphi_{\mathrm{T}}$, respectively. Therefore, the related parameters are calculated as follows.

The speed components of the OS and TS along the X and $\mathrm{Y}$ coordinate axes are determined by Eq. (1).

The relative speed $V_{R}$ of the TS with respect to the OS is determined by Eq. (2)-(4).

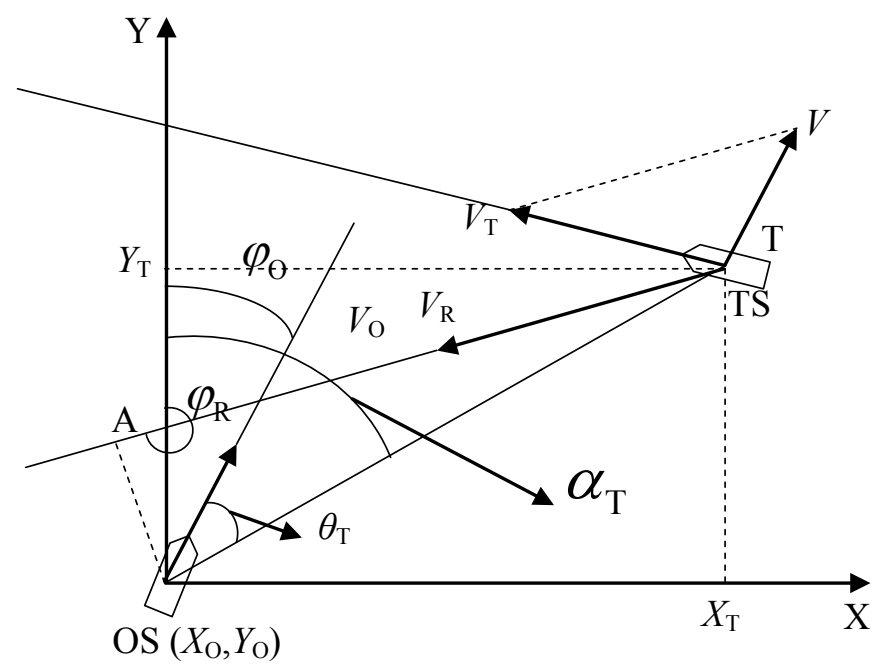

Fig. 1. The space-fixed coordinate system XY 


$$
\begin{gathered}
\left\{\begin{array}{l}
V_{\mathrm{OX}}=V_{\mathrm{O}} \times \sin \left(\varphi_{\mathrm{O}}\right) \\
V_{\mathrm{OY}}=V_{\mathrm{O}} \times \cos \left(\varphi_{\mathrm{O}}\right)
\end{array} \text {. } \begin{array}{l}
V_{\mathrm{TX}}=V_{\mathrm{T}} \times \sin \left(\varphi_{\mathrm{T}}\right) \\
V_{\mathrm{TY}}=V_{\mathrm{T}} \times \cos \left(\varphi_{\mathrm{T}}\right)
\end{array}\right. \\
\left\{\begin{array}{l}
V_{\mathrm{RX}}=V_{\mathrm{TX}}-V_{\mathrm{OX}} \\
V_{\mathrm{RY}}=V_{\mathrm{TY}}-V_{\mathrm{OY}}
\end{array}\right. \\
V_{\mathrm{R}}=\sqrt{V_{\mathrm{RX}}^{2}+V_{\mathrm{RY}}^{2}} \\
\varphi_{\mathrm{R}}=\mathrm{a} \text { tan } \frac{V_{\mathrm{RX}}}{V_{\mathrm{RY}}}+\Delta \alpha \\
\Delta \alpha= \begin{cases}0^{\circ} & \text { if } V_{\mathrm{RX}} \geq 0, V_{\mathrm{RY}}>0 \\
360^{\circ} & \text { if } V_{\mathrm{RX}}<0, V_{\mathrm{RY}}>0 \\
180^{\circ} & \text { others }\end{cases}
\end{gathered}
$$

The relative distance $R_{\mathrm{T}}$ between the OS and the TS is calculated as:

$$
R_{\mathrm{T}}=\sqrt{\left(X_{\mathrm{T}}-X_{\mathrm{O}}\right)^{2}+\left(Y_{\mathrm{T}}-Y_{\mathrm{O}}\right)^{2}}
$$

The true bearing between each other are shown below.

$$
\begin{aligned}
& \alpha_{\mathrm{T}}=\operatorname{atan} \frac{X_{\mathrm{T}}-X_{\mathrm{O}}}{Y_{\mathrm{T}}-Y_{\mathrm{O}}}+\Delta \alpha \\
& \alpha_{\mathrm{O}}=\operatorname{atan} \frac{X_{\mathrm{O}}-X_{\mathrm{T}}}{Y_{\mathrm{O}}-Y_{\mathrm{T}}}+\Delta \alpha
\end{aligned}
$$

The relative bearing of the TS with respect to the OS is displayed below.

$$
\theta_{\mathrm{T}}=\alpha_{T}-\varphi_{\mathrm{O}} \pm 360^{\circ}
$$

The Distance at the Closest Point of Approach between the ships is determined as:

$$
\mathrm{DCPA}=R_{\mathrm{T}} \times \cos \left(\varphi_{\mathrm{R}}-\alpha_{\mathrm{T}}-\pi\right)
$$

As a result, the encounter situation can be deduced by the ship motion parameters, the orientation division of the TS as well as the identification indicators for specific encounter types. For example, first calculate the corresponding parameters in Fig. 1 and determine the region which the TS situates in by the parameter $\theta_{\mathrm{T}}$ shown in Fig. 2 . In fact, the orientation division of the TS is categorized into six sections $\left(\mathrm{P}_{1}, \mathrm{P}_{2}, \mathrm{P}_{3}, \mathrm{P}_{4}, \mathrm{P}_{5}, \mathrm{P}_{6}\right)$ which is based on an interpretation of the COLREGs, navigation habits and good seamanship. The corresponding values of the bearing lines taken clockwise are $[\pi / 8, \pi / 2,5 \pi / 8,11 \pi / 8,3 \pi / 2,15 \pi / 8]$, respectively, which are mainly based on the provision of light's arc of horizon defined in COLREGs, except that the region of $\mathrm{P}_{1}$ is a little larger than the visibility of the masthead light defined in Annex I 9(a) of the COLREGs. This is because the encounter situation is classified from the point of view of coordination collision avoidance as well as the reduction of uncertainties between head-on and other situations [27]. And, the descriptions of every possible encounter type is listed in Tab 1.

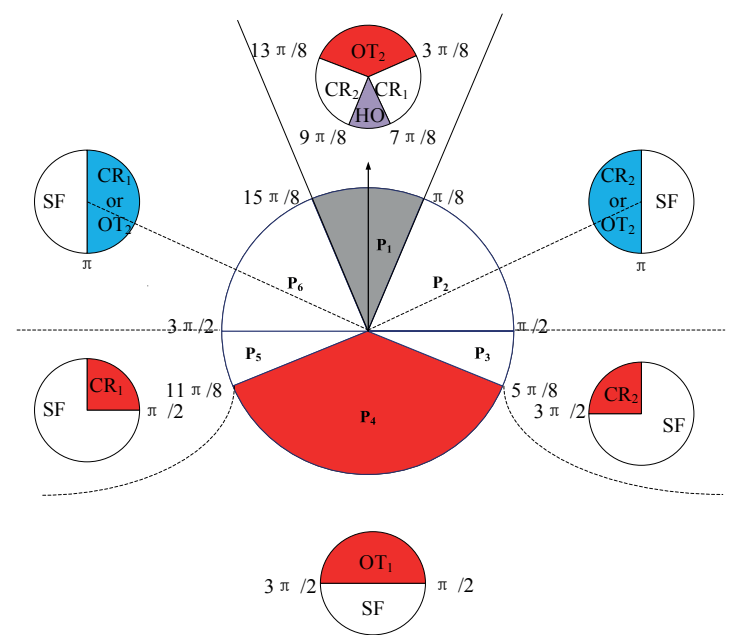

Fig. 2. Orientation division of the TS (the OS is situated at the centre)

Tab. 1. Abbreviations and brief descriptions of the encounter types

\begin{tabular}{|c|l|}
\hline Abbreviations & Description \\
\hline $\mathrm{HO}$ & $\begin{array}{l}\text { Head-on encounter when OS and TS give way } \\
\text { simultaneously }\end{array}$ \\
\hline $\mathrm{OT}_{1}$ & OS is overtaken by TS when TS gives way \\
\hline $\mathrm{OT}_{2}$ & TS is overtaken by OS when OS gives way \\
\hline $\mathrm{CR}_{1}$ & Crossing encounter when TS gives way \\
\hline $\mathrm{CR}_{2}$ & Crossing encounter when OS gives way \\
\hline $\mathrm{SF}$ & Safe encounter \\
\hline
\end{tabular}

Then, the heading of the TS, $\varphi_{\mathrm{T}}$, is roughly divided to judge the possible encounter type. As the target ship can be situated in different regions with respect to the OS, the categorizing parts are different as shown in Fig. 2.

Finally, the encounter type will be determined based on the combination of the mentioned TS's relative bearing, the TS's heading, together with the Distance at the Closest Point of Approach (DCPA). The details are listed in Tab. 2. As a matter of fact, the collision risk borrows the concept of ship domain by comparing the DCPA with the radius of the ship's domain, and $\mathrm{D}_{\mathrm{s}}$ is the radius of ship domain which is a statistical result in the open sea [19]. Usually, the applicable distance for a specific encounter is mainly affected by the visibility distance of lights measured by sight. By the application of modern navigational aids and the principles of good seamanship, the applicable distance is larger. 
Tab. 2. The identification indicators for specific encounter type (DCPA $<D s)$

\begin{tabular}{|c|c|c|c|c|c|}
\hline & $\mathrm{HO}$ & $\mathrm{CR}_{1}$ & $\mathrm{CR}_{2}$ & $\mathrm{OT}_{1}$ & $\mathrm{OT}_{2}$ \\
\hline $\begin{array}{l}\mathrm{P}_{1} \\
\theta_{\mathrm{T}} \in[15 \pi / 8,17 \pi / 8]\end{array}$ & $\left(\varphi_{\mathrm{T}}-\varphi_{0}\right) \in[7 \pi / 8,9 \pi / 8]$ & $\left(\varphi_{\mathrm{T}}-\varphi_{0}\right) \in[3 \pi / 8,7 \pi / 8]$ & $\left(\varphi_{\mathrm{T}}-\varphi_{0}\right) \in[9 \pi / 8,13 \pi / 8]$ & --- & $\left(\varphi_{\mathrm{T}}-\varphi_{0}\right) \in[-3 \pi / 8,3 \pi / 8]$ \\
\hline $\begin{array}{l}\mathrm{P}_{2} \\
\theta_{\mathrm{T}} \in[\pi / 8, \pi / 2]\end{array}$ & --- & -- & $\begin{array}{l}\left(\varphi_{\mathrm{T}}-\varphi_{\mathrm{o}}\right) \in[0, \pi] \\
\alpha_{\mathrm{O}}<\varphi_{\mathrm{T}}+5 / 8 \pi\end{array}$ & -- & $\begin{array}{l}\left(\varphi_{\mathrm{T}}-\varphi_{\mathrm{o}}\right) \in[\pi, 2 \pi] \\
\alpha_{\mathrm{O}}<\varphi_{\mathrm{T}}+11 / 8 \pi\end{array}$ \\
\hline $\begin{array}{l}\mathrm{P}_{3} \\
\theta_{\mathrm{T}} \in[\pi / 2,5 \pi / 8]\end{array}$ & --- & $\cdots$ & $\left(\varphi_{\mathrm{T}}-\varphi_{0}\right) \in[3 \pi / 2,2 \pi]$ & $\cdots$ & -- \\
\hline $\begin{array}{l}\mathrm{P}_{4} \\
\theta_{\mathrm{T}} \in[5 \pi / 8,11 \pi / 8]\end{array}$ & -- & $\cdots$ & --- & $\left(\varphi_{\mathrm{T}}-\varphi_{0}\right) \in[-\pi / 2, \pi / 2]$ & -- \\
\hline $\begin{array}{l}\mathrm{P}_{5} \\
\theta_{\mathrm{T}} \in[11 \pi / 8,3 \pi / 2]\end{array}$ & & $\left(\varphi_{\mathrm{T}}-\varphi_{\mathrm{o}}\right) \in[0, \pi / 2]$ & & & \\
\hline $\begin{array}{l}\mathrm{P}_{6} \\
\theta_{\mathrm{T}} \in[3 \pi / 2,15 \pi / 8]\end{array}$ & & $\begin{array}{l}\left(\varphi_{\mathrm{T}}-\varphi_{\mathrm{o}}\right) \in[0, \pi] \\
\alpha_{\mathrm{O}}<\varphi_{\mathrm{T}}+5 / 8 \pi\end{array}$ & & & $\begin{array}{l}\left(\varphi_{\mathrm{T}}-\varphi_{\mathrm{o}}\right) \in[0, \pi] \\
\alpha_{\mathrm{O}}>\varphi_{\mathrm{T}}+5 / 8 \pi\end{array}$ \\
\hline
\end{tabular}

\section{STRUCTURE OF THE GENETIC ALGORITHM}

Genetic algorithm is a series of search algorithms and optimization technologies in terms of the principles of natural selection inspired by Darwin's theory of evolution (the survival of the fittest). In GA-based approaches, the trajectories are represented as chromosomes and GA features a group of candidate solutions (chromosomes) on the response surface. Through genetic operations such as natural selection, recombination and mutation etc., the trajectories with better fitness are found.

\section{STANDARD GENETIC ALGORITHM}

The previous work using GA in the field of trajectory planning is mainly based on the Standard Genetic Algorithm (SGA), which was first presented by Goldberg in 1989 [4]. And the optimization procedure is mainly handled by three kinds of genetic operators (selection, crossover, mutation) within a single population. The flowchart is given in Fig. 3 together with some descriptions shown below. SGA can be defined by a formula with eight parameters:

$$
S G A=\left(C, P_{0}, M, E, \phi, p_{c}, p_{m}, T\right)
$$

Where $\mathrm{C}$ is the individual encoding method, $P_{0}$ - the initial population, $M$ - the population size, $E$ - the fitness evaluation, $\Phi$ - the selection operator, $p_{c}-$ the crossover operator, $p_{m}-$ the mutation operator, and $T-$ the termination condition.

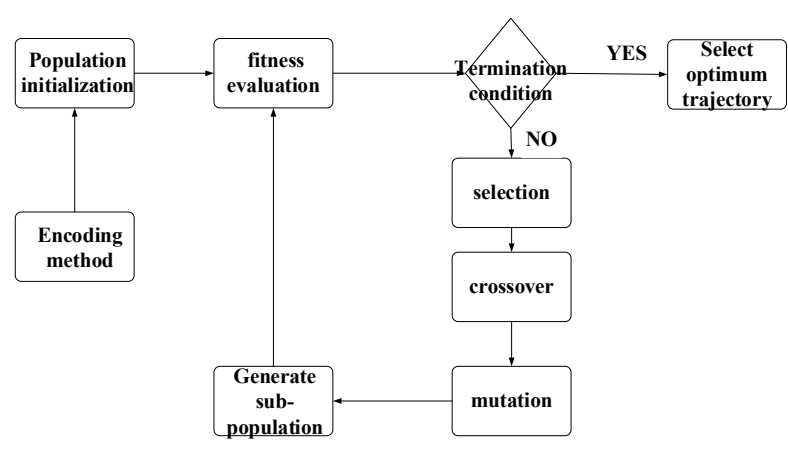

Fig. 3. The flowchart of SGA

\section{MULTIPLE POPULATION GENETIC ALGORITHM}

One of the important configurations in SGA is to determine the parameters' values for genetic operators including the crossover rate $p_{c}$ and the mutation rate $p_{m}$, which both have a great influence on GA's performance. In fact, the previous study usually adopts experiments to determine the parameters for genetic operators when applying SGA for ship's trajectory planning. As a result, it is necessary to dynamically adjust the values of genetic operators to ensure its optimal ability for different traffic scenarios.

Based on the above analysis, the MPGA is adopted and its structure is presented in Fig. 4. The MPGA breaks through the simple population searching frame of SGA, and introduces multiple populations with different combinations of parameters' values to evolve simultaneously. The communication within these populations are closely interwoven by the establishment of an Immigrant Operator, and the main idea is that the worst chromosome in any 
population is replaced by the best chromosome in the adjacent population to achieve co-evolution. Besides, by the establishment of an Elite-individual Operator, the individuals with higher fitness will be chosen to enter the elite population so that the superior individuals will not be lost and make it possible to find the global optimal solution. Furthermore, the elite population does not do selection, crossover or mutation operation for ensuring the integrity of the best individuals.

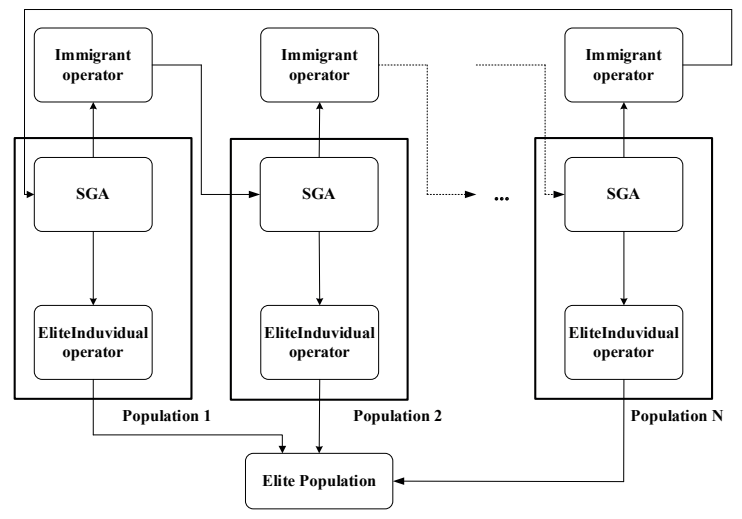

Fig. 4. The flowchart of MPGA

\section{NONLINEAR OPTIMIZATION METHOD}

Nonlinear Programming is a mathematical programming method whose objective function is nonlinear or some of its constraints have nonlinear relationship. It is an important branch of operations research which has wide applications in the field of economics, engineering optimization, management science etc. [1]. As Nonlinear Programming has strong compatibility and robustness in the aspects of local optimization, this work integrates the Nonlinear Programming with MPGA to maintain a balance in the ability of global and local optimization. In fact, the software 'Matlab' provides the multivariable binding optimization function 'fmincon', which attempts to find a constrained minimum of a scalar function of several variables starting at an initial estimate shown below. The basic thought is to search the optimal solution from initial feasible solution, which finds another better one by iteration method based on specific constraints until the optimal one is fond.

$[x, f v a l]=\operatorname{fmincon}\left(f(x), x_{0}, A, b\right.$, Aeq, beq $, l b, u b$, nonlcon ops $)$

$$
\min f(x) \rightarrow\left\{\begin{array}{c}
A x \leq b \\
A_{e q} x=b_{e q} \\
l b<x<u b \\
c(x) \leq 0 \\
c_{e q}(x)=0
\end{array}\right.
$$

Where $x$ is the solution returned as a real vector or array, and ' $\mathrm{f} v \mathrm{al}$ ' is the objective function value at solution. As for the input parameters, $f(x)$ is the objective function, $x$ is the initial point for $x$. Linear constraint matrices $A$ and $A_{e q}$, and their corresponding vectors $b$ and $b_{e q}$, can be sparse or dense. The boundary of $x$ is $l b$ to $u b$. And 'nonlcon' contains the nonlinear equality constraints $c_{e q}(x)=0$ and the nonlinear inequality constraints $c(x) \leq 0$, which the objective function attempts to satisfy. Finally, 'ops' is the optimization options, there are four different optimal algorithms including 'Interiorpoint algorithm', 'Active-set algorithm', 'Sqp algorithm' and 'Turst-region-reflective algorithm'. It is remarkable that 'Interior-point algorithm' handles large, sparse problems, as well as small dense problems; 'Sqp' one satisfies bounds at all iterations and the algorithm can recover from $\mathrm{NaN}$ or Inf results; 'Active-set' one can take large steps, which adds speed; Trust-region-reflective' one requires to provide a gradient, and allows for only bounds or linear equality constraints, but not both.

\section{MODELLING OF HYBRID GENETIC ALGORITHM IN SHIP'S TRAJECTORY PLANNING}

In this paper, a novel hybrid genetic algorithm is proposed for ship's trajectory planning. First, the encounter type is determined according to Section 2. Based on the result, a heuristic method is constructed for the population initialization. Then, by the establishment of the Immigrant Operator and Elite-individual Operator, several populations with different combinations of the parameters' value for genetic operators evolve simultaneously (MPGA). Meanwhile, the adoption of Nonlinear Programming helps enhance the ability of GA in local optimization, and guarantee the optimal solutions satisfy the requirement of practicality with fewer course deviations as well as the consistency. This technique is illustratedin Fig. 5.

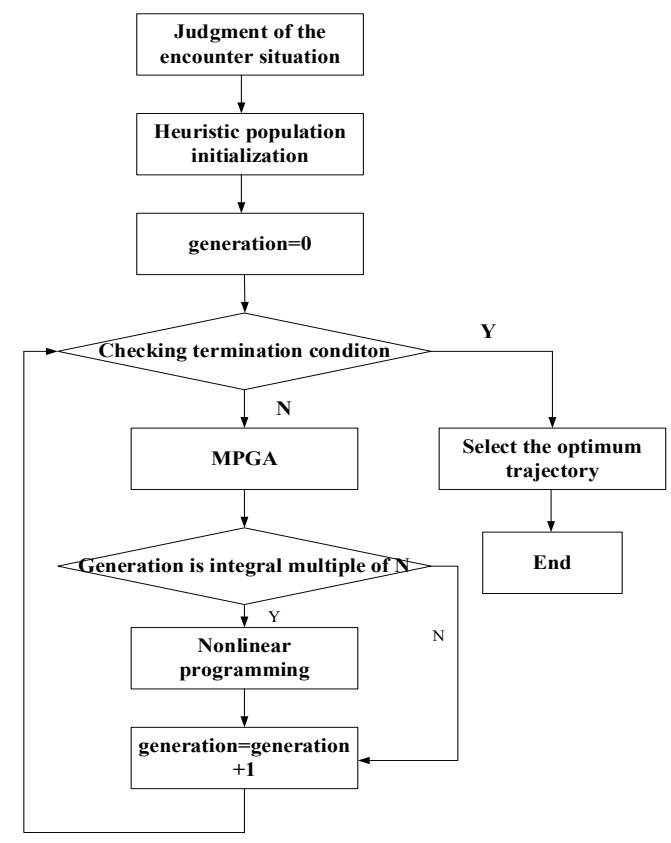

Fig. 5. The flowchart of hybrid genetic algorithm 


\section{THE ENCODING TECHNIQUE}

The encoding technique, the length of code and search space have an influential effect on the optimal speed of the algorithm. As a consequence, the paper adopts the real number encoding method on the basis of previous research, which decreases the dimension of solution space and sets the stage for handling constraint conditions in the Nonlinear Programming. Each chromosome of the population constructed by a series of genes represents an intact trajectory shown in Fig. 6, and each gene represents the heading variation angle between the initial heading and the heading at this turning point.

\begin{tabular}{|l|l|l|l|l|}
\hline$\varphi_{1}$ & $\varphi_{2}$ & $\varphi_{3}$ & $\ldots .$. & $\varphi_{\mathrm{n}}$ \\
\hline
\end{tabular}

Fig. 6. The heading variation angle at different turning points

\section{POPULATION INITIALIZATION}

Compared with random population initialization in the SGA for ship's trajectory planning, this paper adopts a heuristic method to generate initial populations. The idea is to set up constraints in the function of population initialization in accordance with the encounter type and good seamanship. First, to determine the obligation of collision avoidance between ships so that the direction of course changing is decided. If $\varphi_{1}>0$, the obligated ship turns right, otherwise, the obligated ship turns left. Then, considering the intention of evasive manoeuvre should be obvious according to COLREGs, the value of first gene should exceed $15^{\circ}$ [20]. And the deviation of adjacent genes should be less than $\pi / 3$. It's remarkable that the original gene does not do genetic operation in order to satisfy the above-mentioned constraints. In fact, this heuristic method lowers the computational cost in the optimal process. The constraints are calculated as follows:

$$
\varphi_{i} \rightarrow\left\{\begin{array}{c}
\pi / 12<\left|\varphi_{1}\right|>\pi / 6 \\
\|\left(\varphi_{i+1}+\varphi_{1}\right)|-|\left(\varphi_{i}+\varphi_{1}\right)|| \leq \pi / 3(i=2,3, \cdots, N)
\end{array}\right.
$$

\section{FITNESS FUNCTION MODEL}

The fitness evaluation of a chromosome measures the cost of a trajectory, which mainly accommodates three different optimal goals: 1) the safety requirements; 2) less distance traveled; 3 ) the restoration of navigational state. Therefore, we have selected a linear combination of these factors.

$$
f=\left\{\begin{array}{l}
\beta \times\left|D_{\mathrm{e}}\right|+\gamma \times D_{\mathrm{t}} \quad D_{\mathrm{t}}<\mathrm{D}_{\mathrm{s}} \\
\beta \times\left|D_{\mathrm{e}}\right|+\alpha \times\left(D_{\mathrm{t}}-\mathrm{D}_{\mathrm{s}}\right) \quad D_{\mathrm{t}}>\mathrm{D}_{\mathrm{s}}
\end{array}\right.
$$

$$
\begin{gathered}
D_{\mathrm{e}}=\sqrt{\begin{array}{l}
\left(R_{3}(n, 1)-R_{1}(n, 1)\right)^{2}+ \\
\left(R_{3}(n, 2)-R_{1}(n, 2)\right)^{2}
\end{array}} \\
D_{t}=\min \left(\sqrt{\left(R_{3}(t, 1)-R_{2}(t, 1)\right)^{2}+\left(R_{3}(\mathrm{t}, 2)-R_{2}(t, 2)\right)^{2}}\right) \\
(t=0, T, 2 \times T, 3 \times T, \cdots, n \times T)
\end{gathered}
$$

Where $R_{1}, R_{2}, R_{3}$ are two-dimensional coordinate arrays that record the original trajectory of the OS, the trajectory of the TS and the optimal trajectory of the OS. $D_{e}$ is the destination deviation between the optimal trajectory and the original trajectory of the OS. $D_{t}$ is the minimum distance during the movement between the OS and TS. T is the time step. The weighting coefficients of $\alpha$ and $\beta$ indicate the risk of collision and the deviation between the optimal trajectory and the original trajectory of the OS. And $\gamma$ is the penalty factor.

\section{GENETIC OPERATION}

Selection operator: this paper adopts roulette wheel selection for real number encoding method.

$$
p_{i}=\frac{F_{i}}{\sum_{j=1}^{N} F_{j}}
$$

Where $F_{i}$ is the fitness value of chromosome $i$ and $N$ is the total number of chromosomes for each population.

Crossover operator: this paper applies one-point crossover for real number encoding shown as follows:

$$
\begin{aligned}
& a_{k j}=a_{k j}(1-b)+a_{l j} b \\
& a_{l j}=a_{l j}(1-b)+a_{k j} b
\end{aligned}
$$

Where $a_{k j}$ is the value of $j$ '-th gene in the chromosome $k$; $a_{l j}$ - the value of $j$ '-th gene in the chromosome $l ; b$ - a random number between 0 and 1 .

Mutation operator: this paper adopts Gauss mutation operator which generates a random number conforming to normal distribution to replace the value of specific gene.

$$
\begin{aligned}
& a_{i j}=\operatorname{normrnd}(0.5 *(\operatorname{bound}(i, 1)+\operatorname{bound}(i, 2)), \\
& (\operatorname{bound}(i, 2)-\operatorname{bound}(i, 1)) / 6)
\end{aligned}
$$

Where bound $(i, 1)$ is the lower limit of $i$ '-th gene and bound $(i, 2)$ is the upper limit of $i$ '-th gene; the order of 'normrnd' in the Matlab generates random numbers from the normal distribution with mean parameter and standard deviation. 


\section{NONLINEAR OPTIMIZATION}

The application of Nonlinear Programming is used to further tap the potential in the ability of local optimization and improve the superiority of the final solution. It is applied every 50 generations and the detailed constraints are described as follows:

$$
\min \left(f(\varphi)=D_{e}\right) \rightarrow\left[\begin{array}{c}
\varphi_{1}+\varphi_{2} \cdots+\varphi_{\mathrm{n}}=0 \\
\varphi \in\left[-30^{\circ}, 30^{\circ}\right] \\
c(x)=D_{s}-D_{t} \leq 0 \\
c_{e}(\varphi) \in \varnothing
\end{array}\right.
$$

\section{SIMULATION AND ANALYSIS}

Several typical traffic scenarios are adopted for simulation. The encounter type and the obligation are determined based on Fig. 2 and corresponding indicators given in Tab. 2 . The simulation results are primarily intended to evaluate the optimal solutions in the aspects of the consistency and practicality. It is noticeable that the set of test cases are mainly based on target ships approaching the OS from a range of directions. And Tab. 3 shows the initial configuration of the traffic scenarios for all test cases.

Tab. 3. Ship encounter information for different traffic scenarios

\begin{tabular}{|c|c|c|c|c|}
\hline Parameters & Head-on & Crossing & Overtaking & Multi-ship \\
\hline $\begin{array}{c}\text { Position } \\
\text { of OS }(\mathrm{km})\end{array}$ & $(0,0)$ & $(0,0)$ & $(0,0)$ & $(0,0)$ \\
\hline Speed of OS(m/s) & 7.5 & 7.5 & 11 & 7.5 \\
\hline Course of OS $\left(^{\circ}\right)$ & 45 & 45 & 45 & 45 \\
\hline $\begin{array}{c}\text { Position } \\
\text { of TS }(\mathrm{km})\end{array}$ & $(10,10)$ & $(14,6)$ & $(3,3)$ & $(10,10) /(14,6)$ \\
\hline Speed of TS(m/s) & 5 & 7.5 & 5 & $5 / 7.5$ \\
\hline Course of TS $\left(^{\circ}\right)$ & 225 & 270 & 45 & 225 \\
270
\end{tabular}

Let's assume that the own ship's speed is constant. And the OS only relies on changing its course to perform the process of collision avoidance, which is complaint with Rule 8 of the COLREGs for action to avoid a close-quarter situation. After a comprehensive consideration of the traffic scenarios for collision avoidance, the relevant parameters of the algorithm is set as follows: a population size of 3 , a group size of 100 , the termination condition is 100 , and the parameters $\alpha$ and $\beta$ are equal to 0.6 and 0.4 , respectively. In order to contrast the performance of the optimal trajectory, the simulation tests respectively performed by the hybrid genetic algorithm and SGA, adopt the same number of chromosomes. The hybrid genetic algorithm is ran five times in every traffic scenario. The optimal solutions for different test cases are respectively recorded in Tab. 4-7, which remain unchanged for the same input data. The corresponding curves of course variation, optimal trajectory and distance variation are shown in Fig. 7-9 (head-on), Fig. 10-12 (crossing), Fig. 13-15 (overtaking) and Fig. 16-18 (multitarget), accordingly. The evolutionary process is described in Fig. 19. In the trajectory curves, black line and black dash dot line respectively represent the optimal trajectory of the OS obtained by using the proposed algorithm and SGA, blue line indicates the trajectory of the TS, red dotted line indicates the original trajectory of the OS. The information about DCPA and the corresponding time (TCPA) are clearly marked in the distance variation curves between OS and TS.

\section{HEAD-ON SITUATION}

In this encounter situation, the TS comes towards the OS from the region $\mathrm{P}_{1}$, and the corresponding parameters meets the requirement for $\mathrm{HO}$ given in Tab. 1. Meanwhile, the ship domain will be violated with the approaching of the TS. As a consequence, this encounter type can be interpreted by Rule 14 of the COLREGs, which indicates that the ships should pass each other port to port. It is remarkable that the evasive manoeuvre is conducted from the perspective of the OS. And the action taken by the OS guarantees the safety though the TS does not take any action. The course variation, the optimal trajectory and distance variation are presented in Fig. 7-Fig. 9.

Tab.4. Optimal solutions in head-on situation

\begin{tabular}{|c|c|c|c|c|c|}
\hline Sequence & $\varphi_{1}\left({ }^{\circ}\right)$ & $\varphi_{2}\left({ }^{\circ}\right)$ & $\varphi_{3}\left({ }^{\circ}\right)$ & $\varphi_{4}\left({ }^{\circ}\right)$ & $\varphi_{5}\left({ }^{\circ}\right)$ \\
\hline Trajectory1 & 20 & 14 & 14 & 14 & -10 \\
\hline Trajectory2 & 20 & 14 & 14 & 14 & -10 \\
\hline Trajectory3 & 20 & 14 & 14 & 14 & -10 \\
\hline Trajectory4 & 20 & 14 & 14 & 14 & -10 \\
\hline Trajectory5 & 20 & 14 & 14 & 14 & -10 \\
\hline
\end{tabular}

Continuation of Tab. 4: Optimal solutions in head-on situation

\begin{tabular}{|c|c|c|c|c|c|}
\hline Sequence & $\varphi_{6}\left({ }^{\circ}\right)$ & $\varphi_{7}\left({ }^{\circ}\right)$ & $\varphi_{8}\left({ }^{\circ}\right)$ & $\varphi_{9}\left(^{\circ}\right)$ & $\varphi_{10}\left({ }^{\circ}\right)$ \\
\hline Trajectory1 & -10 & -10 & -10 & -10 & -10 \\
\hline Trajectory2 & -10 & -10 & -10 & -10 & -9 \\
\hline Trajectory3 & -10 & -10 & -10 & -10 & -10 \\
\hline Trajectory4 & -10 & -10 & -10 & -10 & -10 \\
\hline Trajectory5 & -10 & -10 & -10 & -10 & -9 \\
\hline
\end{tabular}

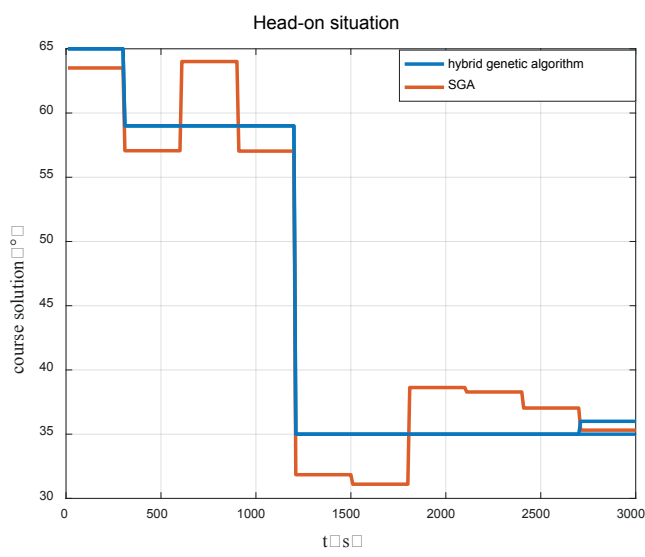

Fig. 7. The course variation of the optimal trajectory 


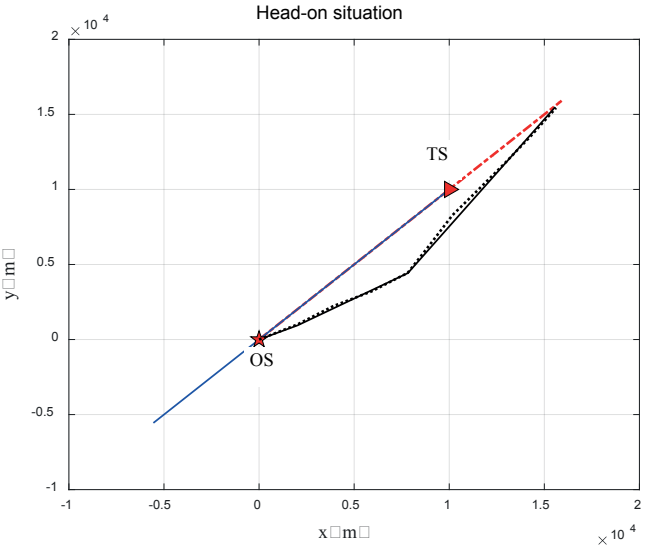

Fig. 8. Optimal trajectory in head-on situation

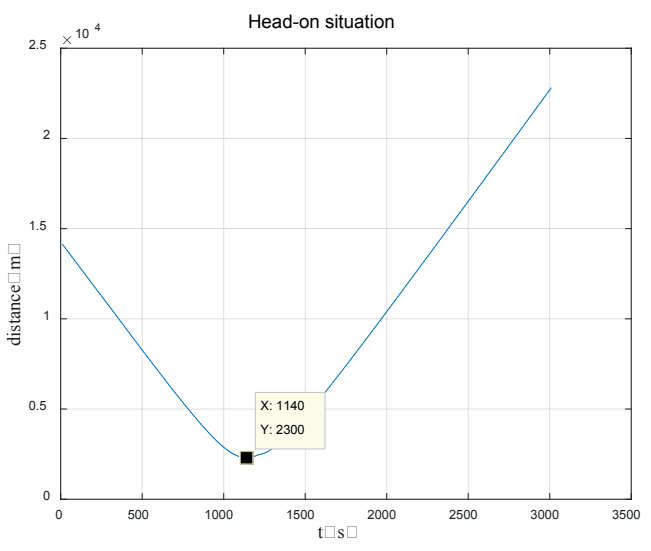

Fig. 9. Distance variation curve in head-on situation

\section{CROSSING SITUATION}

In this encounter situation, the TS comes towards OS from region $P_{2}$. Similarly, this encounter type allows to evaluate the collision risk interpreting Rule 15 of the COLREGs for a crossing situation. It is deemed practical to pass TS on its stern. The corresponding course variation, optimal trajectory and distance variation are shown in Fig. 10-Fig. 12.

Tab. 5. Optimal solutions in crossing situation

\begin{tabular}{|c|c|c|c|c|c|}
\hline Sequence & $\varphi_{1}\left({ }^{\circ}\right)$ & $\left.\varphi_{2}{ }^{\circ}\right)$ & $\varphi_{3}\left({ }^{\circ}\right)$ & $\varphi_{4}\left({ }^{\circ}\right)$ & $\varphi_{5}\left({ }^{\circ}\right)$ \\
\hline Trajectory1 & 19 & 15 & 15 & 15 & -10 \\
\hline Trajectory2 & 19 & 15 & 15 & 15 & -10 \\
\hline Trajectory3 & 19 & 15 & 15 & 15 & -10 \\
\hline Trajectory4 & 19 & 15 & 15 & 15 & -10 \\
\hline Trajectory5 & 19 & 15 & 15 & 15 & -10 \\
\hline
\end{tabular}

Continuation of Tab. 5: Optimal solutions in crossing situation

\begin{tabular}{|l|c|c|c|c|c|}
\hline Sequence & $\varphi_{6}\left({ }^{\circ}\right)$ & $\varphi_{7}\left({ }^{\circ}\right)$ & $\varphi_{8}\left({ }^{\circ}\right)$ & $\varphi_{9}\left({ }^{\circ}\right)$ & $\varphi_{10}\left({ }^{\circ}\right)$ \\
\hline Trajectory1 & -10 & -10 & -10 & -10 & -10 \\
\hline Trajectory2 & -10 & -10 & -10 & -10 & -10 \\
\hline Trajectory3 & -10 & -10 & -10 & -10 & -10 \\
\hline Trajectory4 & -10 & -10 & -10 & -10 & -10 \\
\hline Trajectory5 & -10 & -10 & -10 & -10 & -10 \\
\hline
\end{tabular}

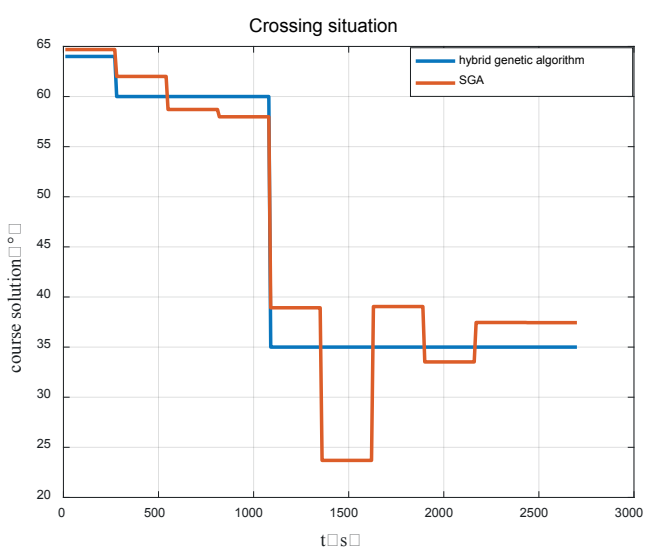

Fig. 10. The course variation of the optimal trajectory

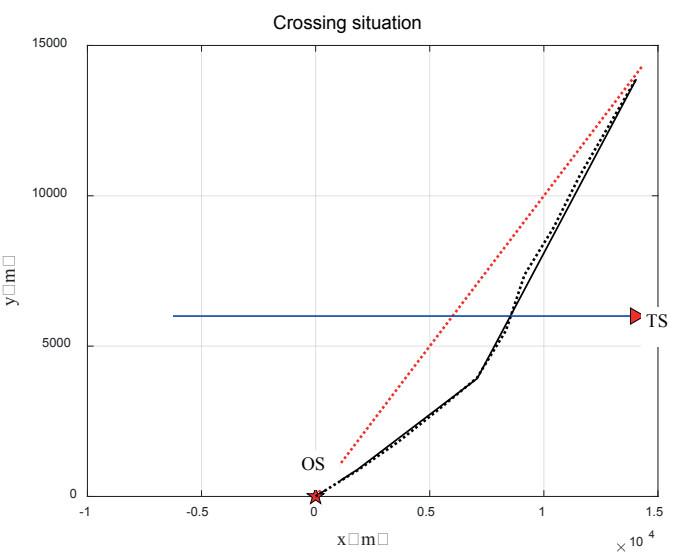

Fig. 11. Optimal trajectory in crossing situation

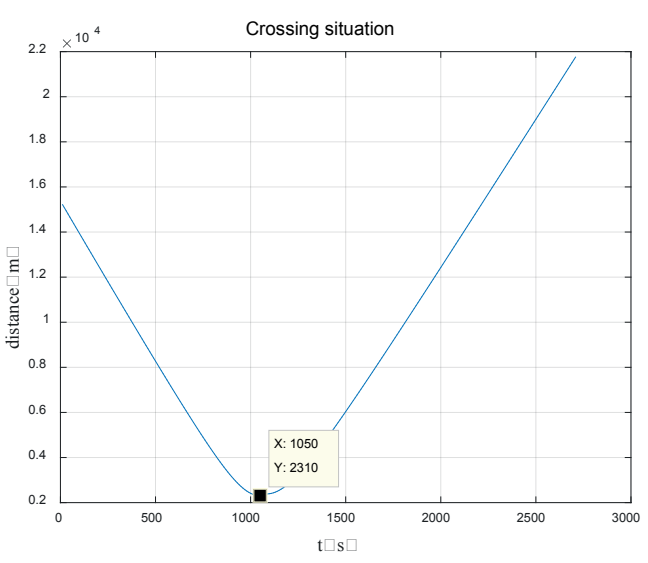

Fig. 12. Distance variation curve in crossing situation

\section{OVERTAKING}

In this situation, the OS overtakes the TS from the stern of the target when the TS is situated in region $\mathrm{P}_{1}$. The aim of this test is to evaluate the method's interpretation of Rule 13 of the COLREGs that the OS should give way to the overtaken 
ship. Similarly to head-on and crossing simulation, the key variable curves are described in Fig. 13-Fig.15.

Tab. 6. Optimal solutions in overtaking situation

\begin{tabular}{|c|c|c|c|c|c|}
\hline Sequence & $\varphi_{1}\left({ }^{\circ}\right)$ & $\varphi_{2}\left({ }^{\circ}\right)$ & $\varphi_{3}\left({ }^{\circ}\right)$ & $\varphi_{4}\left({ }^{\circ}\right)$ & $\left.\varphi_{5}{ }^{\circ}\right)$ \\
\hline Trajectory1 & 22 & 18 & 12 & -7 & -7 \\
\hline Trajectory2 & 22 & 18 & 12 & -7 & -7 \\
\hline Trajectory3 & 22 & 18 & 12 & -7 & -7 \\
\hline Trajectory4 & 22 & 18 & 12 & -7 & -7 \\
\hline Trajectory5 & 22 & 18 & 12 & -7 & -7 \\
\hline
\end{tabular}

Continuation of Tab. 6: Optimal solutions in overtaking situation

\begin{tabular}{|c|c|c|c|c|c|}
\hline Sequence & $\left.\varphi_{6}{ }^{(}\right)$ & $\varphi_{7}\left({ }^{\circ}\right)$ & $\varphi_{8}\left({ }^{\circ}\right)$ & $\varphi_{9}\left({ }^{\circ}\right)$ & $\varphi_{10}\left({ }^{\circ}\right)$ \\
\hline Trajectory1 & -7 & -7 & -7 & -7 & -7 \\
\hline Trajectory2 & -7 & -7 & -7 & -7 & -7 \\
\hline Trajectory3 & -7 & -7 & -7 & -7 & -8 \\
\hline Trajectory4 & -7 & -7 & -7 & -7 & -8 \\
\hline Trajectory5 & -7 & -7 & -7 & -7 & -7 \\
\hline
\end{tabular}

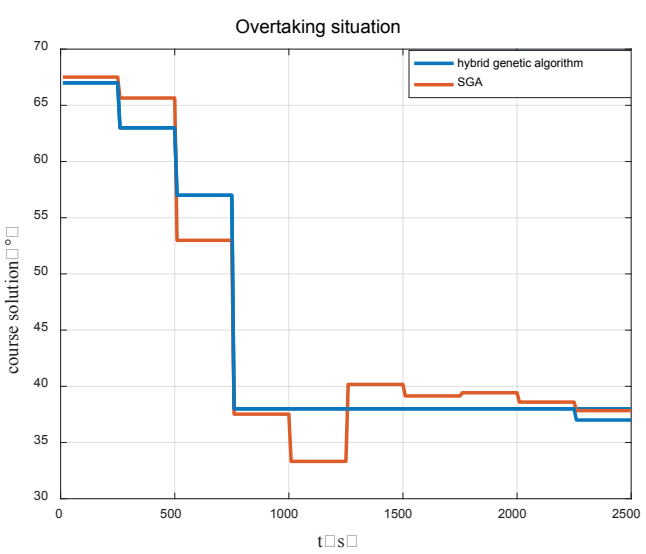

Fig. 13. The course variation of the optimal trajectory

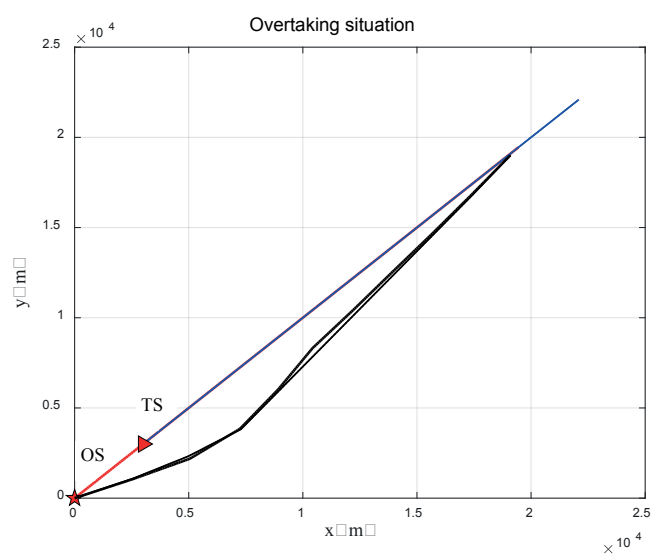

Fig. 14. Optimal trajectory in overtaking situation

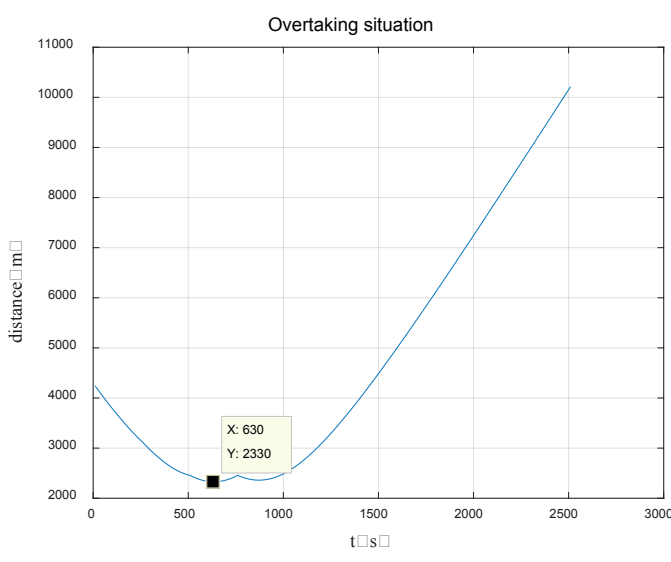

Fig. 15. Distance variation curve in overtaking situation

\section{MULTI-SHIP ENCOUNTER SITUATION}

In this situation, the OS is in a complex condition which faces the encounter types of head-on and crossing at the same time. As there is no risk between target ships shown in Fig. 13, the OS is the only manoeuvring party to perform evasive actions. Therefore, the overall output for the multiship encounter situation is illustrated in Fig. 16 - Fig.18.

Tab. 7. Optimal solutions in multi-ship encounter situation

\begin{tabular}{|c|c|c|c|c|c|}
\hline Sequence & $\left.\varphi_{1}{ }^{\circ}\right)$ & $\varphi_{2}\left({ }^{\circ}\right)$ & $\varphi_{3}\left({ }^{\circ}\right)$ & $\varphi_{4}\left({ }^{\circ}\right)$ & $\left.\varphi_{5}{ }^{\circ}\right)$ \\
\hline Trajectory1 & 21 & 14 & 14 & 14 & -10 \\
\hline Trajectory2 & 21 & 14 & 14 & 14 & -10 \\
\hline Trajectory3 & 21 & 14 & 14 & 14 & -10 \\
\hline Trajectory4 & 21 & 14 & 14 & 14 & -10 \\
\hline Trajectory5 & 21 & 14 & 14 & 14 & -10 \\
\hline
\end{tabular}

Continuation of Tab. 7: Optimal solutions in multi-ship encounter situation

\begin{tabular}{|c|c|c|c|c|c|}
\hline Sequence & $\varphi_{6}\left({ }^{\circ}\right)$ & $\varphi_{7}\left({ }^{\circ}\right)$ & $\varphi_{8}\left({ }^{\circ}\right)$ & $\varphi_{9}\left({ }^{\circ}\right)$ & $\varphi_{10}\left({ }^{\circ}\right)$ \\
\hline Trajectory1 & -10 & -10 & -10 & -10 & -10 \\
\hline Trajectory2 & -10 & -10 & -10 & -10 & -10 \\
\hline Trajectory3 & -10 & -10 & -10 & -10 & -10 \\
\hline Trajectory4 & -10 & -10 & -10 & -10 & -10 \\
\hline Trajectory5 & -10 & -10 & -10 & -10 & -10 \\
\hline
\end{tabular}

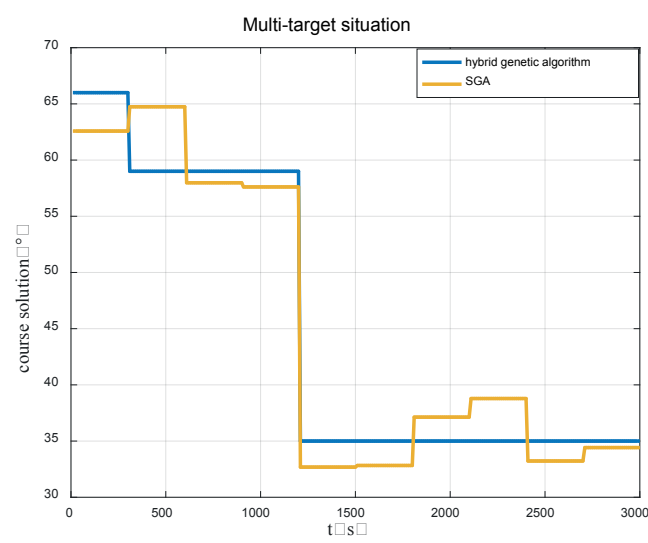

Fig. 16. The course variation of the optimal trajectory 


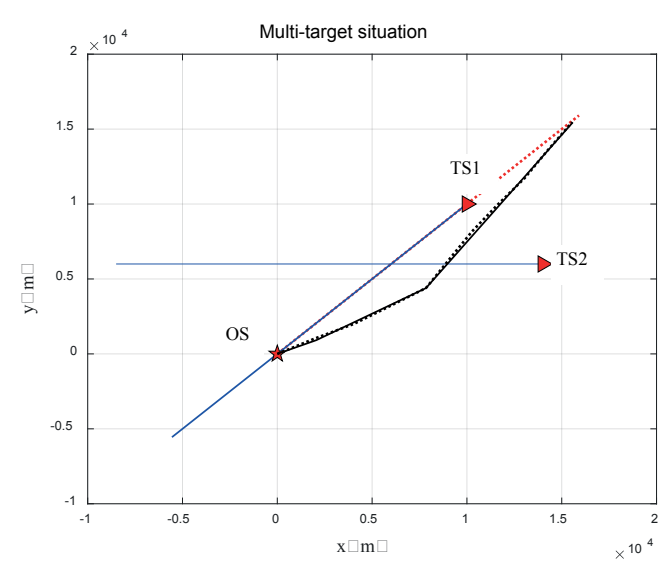

Fig. 17. Optimal trajectory in multi-ship encounter situation

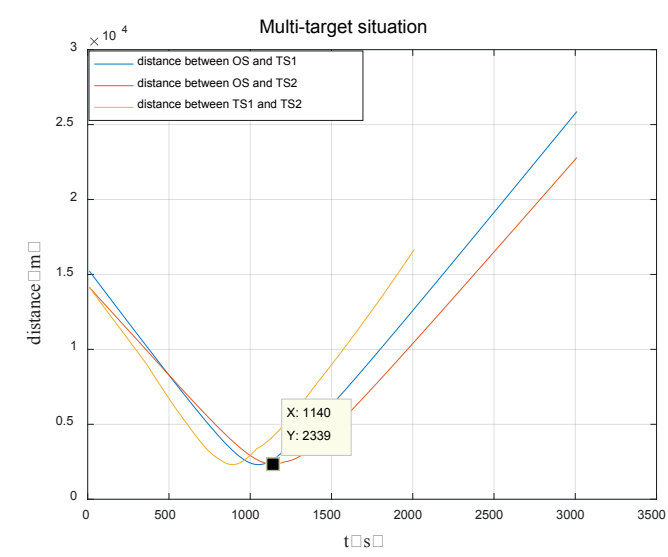

Fig. 18 Distance variation curve in multi-ship encounter situation

\section{EVOLUTIONARY PROCESS}

In order to verify the superiority, we run the hybrid genetic algorithm and SGA with the same number of chromosomes to compare as to fitness value. The convergence process in different traffic scenarios is shown in Fig. 19.

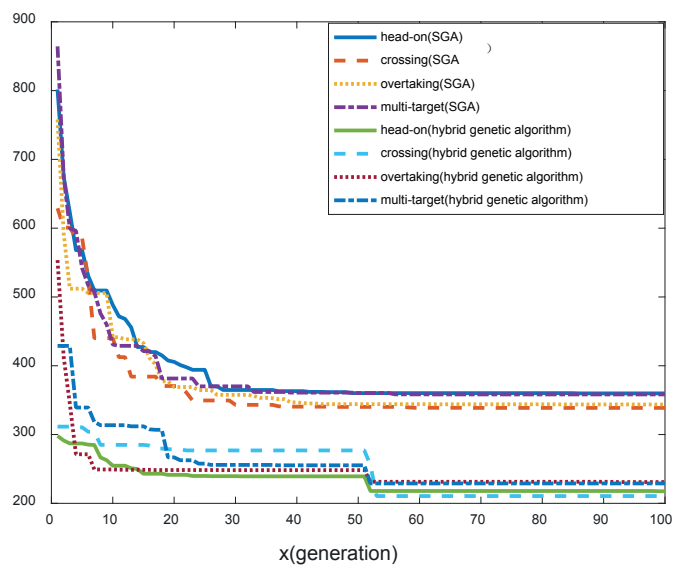

Fig. 19. The evolutionary process for calculation of fitness value

\section{DISCUSSION}

From the simulation results, some conclusions can be drawn. Firstly, the minimum distance between ships (DCPA) in different traffic scenarios exceeds the safety criterion-radius of ship domain $\left(D_{s}\right)$, which meets the safety requirement in the fitness function model. Furthermore, the uniform variation in the relative distance as well as the closeness between DCPA and the radius of ship domain, reflected in the total length of optimal trajectory, is relatively short, which guarantees the distance requirement. Meanwhile, the optimal trajectories in different traffic scenarios contain intact evasive manoeuvres, including the action of collision avoidance and the restoration to the original trajectory. Additionally, the consistency of the evasive manoeuvres is mainly reflected in two aspects. One is that the obligation is certain as the encounter type is derived from COLREGs whether from the position of the OS or the TS, the other is that the optimal solutions presented in each Table (Tab. 4-7) are nearly the same, and the corresponding optimal trajectories and distance variation curves almost overlap. More importantly, it is clear in the course variation curves that the evasive manoeuvres simulated by the hybrid genetic algorithm is performed with fewer course deviations even when multiple ships are involved, compared with the manoeuvres by SGA. This makes the optimal solution more suitable for the common practice of navigators. As for the searching ability, the fitness values in Fig. 19 reflects the better convergence effect by the hybrid genetic algorithm. Meanwhile, there is an obvious leap in the $50^{\text {th }}$ generation which reflects the influence of the Nonlinear Programming on the ability of local optimization. As a result, we may draw the conclusion that the output of the hybrid genetic algorithm is improved in the aspects of consistency and practicality than that of SGA, i.e. demonstrates the feasibility and superiority of the hybrid genetic algorithm.

\section{CONCLUSION}

A novel trajectory planning based on hybrid genetic algorithm has been presented. And several traffic scenarios have been set up for verifying the feasibility and superiority of this proposed algorithm. By comparing the results of the hybrid genetic algorithm and SGA in the aspect of the optimal trajectory, course variation, distance variation as well as the evolutionary process, we can conclude that the hybrid genetic algorithm this hybrid genetic algorithm greatly improves the searching ability and guarantees the optimal solution unchanged for the same input data. Moreover, the final solution with fewer course deviations is more suitable for the practical application.

However, this paper does not consider the influence of ship manoeuvrability as it only discuss the easy multi-ship encounter situation. Therefore, further research plan is aimed at solving the above mentioned issues and establishing the feedback mechanism for monitoring the ship's operational state, which helps to form an intact decision support system. 
Meanwhile, we should try to apply Pareto-based multiobjective optimization method and other selection operators for improving the efficiency of the algorithm.

\section{ACKNOWLEDGEMENTS}

This work is supported by National Natural Science Foundation of China under Grant No. 51309041.

\section{BIBLIOGRAPHY}

1. Bazarra M.S., Shetty L.M.: Nonlinear Programming: Theory and Algorithms. John Wiley and Sons, New York, 1979, pp. 1-360.

2. Benjamin M. R., Curcio J. A.: COLREGS-based navigation of autonomous marine vehicles. Autonomous Underwater Vehicles IEEE/OES. IEEE, 2004, pp. 32-39.

3. Burmeister, H.C., Bruhn W. and Porathe T.: Autonomous unmanned merchant vessel and its contribution towards the e-navigation implementation. the MUNIN perspective. Ocean Engineering. 1, 2014, pp. 1-13.

4. Goldberg D.: Genetic Algorithms in Search, Optimization and Machine Learning, Addison Wesley, Reading, MA, 1989

5. Hong X., Harris C. J., Wilson P. A.: Autonomous ship collision free trajectory navigation and control algorithms. Proceedings of $7^{\text {th }}$ IEEE International Conference on Emerging Technologies and Factory Automation, 1999, pp. 923-929.

6. Hwang C. N., Yang J. M., Chiang C. Y.: The design of fuzzy collision-avoidance expert system implemented by $\mathrm{H}$ (subscript $\infty$ )-autopilot. Journal of Marine Science and technology, 9(1), 2001, pp. 25-37.

7. Hwang C.N.: The integrated design of fuzzy collisionavoidance and h1- autopilots on ships. The Journal of Navigation, 55 (1), 2002, pp.117-136.

8. Ito M., Zhang F. and Yoshida N.: Collision avoidance control of ship with genetic algorithm. Proceedings of 1999 IOOO international conference on control applications, 1999. pp. 1791-1796.

9. Jokioinen E.: Remote and autonomous ships: the next steps. www.rolls-royce.com/marine, 2016

10. Lazarowska A.: Ship's trajectory planning for collision avoidance at sea based on ant colony optimization. Journal of Navigation, 68(2), 2015, pp. 291-307.

11. Lee Y., Kim Y. G.: A collision avoidance system for autonomous ship using fuzzy relational products and
COLREGs. Proceedings of Intelligent Data Engineering and Automated Learning-IDEAL: $5^{\text {th }}$ International Conference, Exeter, UK. 25-27 August, 2004, pp. 247-252.

12. Li, L.N., Yang S.H., Cao B.G. and Li, Z.F.: A summary of studies on the automation of ship Collision Avoidance Intelligence. Journal of Jimei University, China, 11(2), 2006, pp. 199-192.

13. Liu, Y., Bucknall R.: Path planning algorithm for unmanned surface vehicle formations in a practical maritime environment. Ocean Engineering, 97, 2015, pp. 126-144.

14. Miele, A., Wang T., Chao C. S., et al.: Optimal control of a ship for collision avoidance maneuvers. Journal of Optimization Theory and Applications, 103(3), 1999, pp. 495-519.

15. Perera L. P., Carvalho J. P., Soares C. G.: Fuzzy logic based decision making system for collision avoidance of ocean navigation under critical collision conditions. Journal of Marine Science and Technology, 16(1), 2011, pp. 84-99.

16. Perera L. P., Carvalho J. P., Soares C. G.: Intelligent ocean navigation and fuzzy-bayesian decision action formulation. IEEE Journal of Oceanic Engineering, 37(2), 2012, pp. 204-219.

17. Smierzchalski R.: Evolutionary trajectory planning of ships in navigation traffic areas. Journal of Marine Science and Technology. 4(1), 1999, pp. 1-6.

18. Statheros T., Howells G. and McDonald-Maier K.: Autonomous ship collision avoidance navigation concepts, technologies and techniques. Journal of Navigation, 61, 2008, pp. 129-142.

19. Sun L. C.: The Model of close quarter situation. Proceedings of 96'ANTI-COLLISION, 1996.

20. Szlapczynski, R.: A unified measure of collision risk derived from the concept of a ship domain. Journal of Navigation. 59, 2006, pp. 477-490.

21. Szlapczynski, R.. A new method of ship routing on raster grids, with turn penalties and collision avoidance. Journal of Navigation, 59(1), 2006, pp. 27-42.

22. Szlapczynski, R.: Evolutionary sets of safe ship trajectories: a new approach to collision avoidance. Journal of Navigation, 64(1), 2011, pp.169-181.

23. Szlapczynski R., Szlapczynska J.: On evolutionary computing in multi-ship trajectory planning. Applied Intelligence, 37(2), 2012, pp. 155-174. 
24. Szlapczynski R., Szlapczynska J.: Customized crossover in evolutionary sets of safe ship trajectories. International Journal of Applied Mathematics and Computer Science, 22(4), 2012, pp. 999-1009.

25. Szłapczynski R.: Evolutionary sets of safe ship trajectories with speed reduction manoeuvres within traffic separation schemes. Polish Maritime Research, 21(1), 2014, pp. 20-27.

26. Tam C., Bucknall R., Greig A.: Review of collision avoidance and path planning methods for ships in close range encounters. Journal of Navigation. 62, 2009, pp. 455-476.

27. Tam C., Bucknall R.: Collision risk assessment for ships. J. Mar. Sci. Technol. 15, 2010, pp. 257-270.

28. Tam C. K., Bucknall R.: Path-planning algorithm for ships in close-range encounters. Journal of Marine Science and Technology, 15(4), 2010, pp. 395-407.

29. Tam C. K., Bucknall R.: Cooperative path planning algorithm for marine surface vessels. Ocean Engineering, 57, 2013, pp. 25-33.

30. Tsou M.C., Hsueh C.K.: The study of ship collision avoidance route planning by ant colony algorithm. Journal of Marine Science and Technology, 2010, 18(5), 2010, pp. 746-756.

31. Tsou, M. C., Kao S. L., Su C. M.: Decision support from genetic algorithms for ship collision avoidance route planning and alerts. Journal of Navigation, 63(1), 2010, pp. 167-182.

32. Tsou M.C.: Multi-target collision avoidance route planning under an ECDIS framework. Ocean Engineering. 121, 2016, pp. 268-278.

33. Xu Q. Y.: Collision avoidance strategy optimization based on danger immune algorithm. Computers \& Industrial Engineering, 76, 2014, pp. 268-279.

34. Xue Y., Clelland D., Lee B. S., et al.: Automatic simulation of ship navigation. Ocean Engineering, 38(17), 2011, pp. $2290-2305$.

35. Zhang J, F., Zhang D., Yan X., et al.: A distributed anticollision decision support formulation in multi-ship encounter situations under COLREGs. Ocean Engineering, 105, 2015, pp. 336-348.

\section{CONTACT WITH THE AUTHORS}

\author{
Shengke Ni \\ e-mail:863369021@qq.com \\ Dalian Maritime University \\ Linghai road \\ 116026 Dalian \\ China
}

\section{Zhengjiang Liu}

e-mail:2522402892@qq.com

Dalian Maritime University

Linghai road

116026 Dalian

China

Yao Cai

e-mail:3492105068@qq.com

Dalian Maritime University

Linghai road

116026 Dalian

China

\author{
Xin Wang \\ e-mail:875924999@qq.com \\ Dalian Maritime University \\ Linghai road \\ 116026 Dalian
}

China 